\title{
Téoros
}

Revue de recherche en tourisme

\section{Avenirs de la villégiature balnéaire en France : quelques pronostics à propos des vacanciers du littoral...}

\section{Jean-Didier Urbain}

Volume 12, numéro 2, juillet 1993

La prévision-prospective du tourisme

URI : https://id.erudit.org/iderudit/1078025ar

DOI : https://doi.org/10.7202/1078025ar

Aller au sommaire du numéro

Éditeur(s)

Université du Québec à Montréal

ISSN

0712-8657 (imprimé)

1923-2705 (numérique)

Découvrir la revue

Citer cet article

Urbain, J.-D. (1993). Avenirs de la villégiature balnéaire en France : quelques pronostics à propos des vacanciers du littoral... Téoros, 12(2), 40-43.

https://doi.org/10.7202/1078025ar 


\section{Avenirs de la villégiature balnéaire en France : quelques pronostics à propos des vacanciers du littoral... Jean-Didier Urbain ${ }^{*}$}

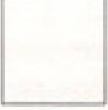

Entre opinions et statistiques, attitudes et comportements, la prospective des vacances et des loisirs est tout particulièrement mise à l'épreuve à l'occasion de l'analyse de l'évolution des séjours balnéaires.

D'une part, cette prospective est en effet soumiseà la pression de rumeurs persistantes qui annoncent des progrès sensibles de fréquentation en milieux rural et montagnard. Ainsi, chaque été, les médias ne manquent pas d'évoquer le renouveau du stourisme verts à l'aide de quelques reportages convaincants sur la randonnée, artisanat et vacanciers ou l'agro-tourisme.

À cela s'ajoute la publication d'indicateurs de fiabilité fortrelative tant les estimations varient. Ainsi, par exemple, l'on peut lire dans cet ouvrage, pour l'été 1991: *Les séjoursà la campagne età la montagne sont en légère progression; ils représentent respectivement $22 \%$ et $13 \%$ des séjours (i) $^{(1)}$. Or, pour l'année 1990 et d'après le ministère du Tourisme ${ }^{(2)}$, la campagne représentait déjà $23,4 \%$ des séjours estivaux et la montagne $14,9 \%$ ! Qu'en est-il alors de ladite elégère progressions qui, confrontée à ces autres indicateurs, fait maintenant figure de régression?

D'autre part, cette prospective se trouve confrontée à des données quantitatives inscrites dans la longue durée - et de ce fait plus fiables, en ce qu'elles attestent au moins d'une tendance pérenne, en dépit de leurs fluctuations. Ainsi, l'on constate sur trente ans la constante progression du goût des Français pour la villégiature balnéaire passée de $36 \%$ en 1961 à près de $49 \%$ en 1990 -, une progression que, par contraste, confirme cruellement la chute corrélative et non moins constante de la fréquentation vacancière de la campagne et de la montagne en été.

Voici donc, sur un quart de siècle, quelques chiffres concernant la répartition (en \%) des séjours de vacances d'été en France selon le genre de séjour. Ils résument les tendances de fréquentation contraires dela mer, d'une part, et de la montagne et de la campagne, d'autre part - chiffres auxquels sont adjoints pour information ceux de la ville et du circuit, conformément aux catégories retenues par l'INSEE (tableau 1).

Cela suffit, semble-t-il, à démontrer la force d'attraction considérable qu'exerce le littoral sur la population vacanciöre = une force qui, quoique pondérée par d'autres activités davantage privilégiées hors des frontières (ville et circuit), n'est pas démentie lors des vacances à l'étranger, mêmesi la campagne est là, en effet, en progression sensible (tableau 2).

Autrement dit, voici notre prospective déchirée entre, concernant montagne et campagne, quelques indicateurs encore faibles pour être totalement significatifs et la réalité incontestable d'une tendance lourde tournée vers le littoral. Ces indicateurs alimentent une espérance récurrente qui n'est, à bien des égards, qu'une prophétie optimiste cachant mal le plus souvent une demande dérue de développenent ou de redéploienent du *tourime intárieurs - et cela en dépit des sports d'hiver pour l'une et de l'espoir d'une clientèle internationale, allemande notamment, pour l'autre ${ }^{(3)}$.

En fait, il faut que l'hiver arrive pour que, en France, la campagne repasse en tête des séjours, avec $29,8 \%$ en 1990 , et renoue ainsi avec un pourcentage de fréquentation qu'elle avait encore à la fin des années 1960 et au début des années 1970, en été. De même pour la montagne, qui, grâce aux sports d'hiver, se maintient en seconde position, en dépit d'un déclin sensible ces dernières années, étant passće de $25,7 \%$ en 1988 à $21,8 \%$ en 1990 . Corrélativement, on notera que la mer fait mieux que résister durant la mème période, puisqu'elle est passće de $17,6 \%$ à $19,6 \%$ des séjours et qu'au nombre de journées elle dépasse la montagne avec $20,6 \%$ contre $19,3 \%$. Quant aux séjours à la montagne en 1990 , ils sont, hors sports d'hiver, en légère progression, étant passés de $8,9 \%$ en 1988 à $9,9 \%$ en 1990.

Notons au passage que cette propension vacancière pour le littoral ne semble pas 
TABLEAU 1 :

\begin{tabular}{lrrrrrr}
\hline & Mer & Montagne & Campagne & Ville & Circuit & Total \\
\hline 1965 & 39,0 & 19,5 & 34,5 & 4,5 & 2,5 & 100 \\
1975 & 43,3 & 16,9 & 25,3 & 7,3 & 7,2 & 100 \\
1985 & 44,6 & 15,6 & 24,1 & 7,9 & 7,8 & 100 \\
1990 & 48,9 & 14,9 & 23,4 & 8,1 & 4,7 & 100 \\
\hline
\end{tabular}

TABLEAU 2:

\begin{tabular}{lllllll}
\hline 1988 & 38,3 & 7,5 & 14,7 & 18,0 & 21,5 & 100 \\
1990 & 37,0 & 7,0 & 18,1 & 16,6 & 21,3 & 100 \\
\hline
\end{tabular}

spécifiquement française et que, par exemple, l'on constate la même tendance en Grèce, où, après une période de tourisme culturel tourné vers l'intérieur (entre 1950 et 1965), l'on a assisté à une inversion de tendance en faveur de la villégiature balnéaire entre 1965 et 1980 - une tendance qui a aujourd'hui de fortes chances de s'amplifier encore avec le développement du tourisme national ${ }^{(4)}$.

Il apparait alors nécessaire, afin de se prononcer sur le sens et l'avenir de ces progrès et déclins vacanciers, de plus en plus nettement territorialisés - qui voient s'imposer comme forme vacancière dominante ce que l'on pourrait appeler un *tourisme extérieurs ou spériphériques -, de faire intervenir d'autres paramètres d'analyse, non seulement statistiques mais également davantage qualitatifs, de nature psychosociologique, voire culturelle et anthropologique.

En effet, à quoi correspond au fond: à quel besoin ou à quelle demande, cet attrait pour le bord de mer - ce signed'époque qui se caractérise, comme nombre de sociologues l'ont souligné, par une perted'intérêt chez levacancier pour la vielocale, l'identité du pays, la tradition ou l'authenticité au profit d'un espace vide culturellement démarqué, isoléet essentiellement consacré à un type de résidence enclavée qui, une fois sur place, n'invite que fort peu, voire pas du tout, à la mobilité, à l'exploration de l'arrière-pays, au contact ou même à la simple excursion?

Nul doute qu'il y a là, au niveau de l'anthropologie des vacances et du tourisme, une réalité dont l'ampleur (sinon la nousoi... d'itinérance et voyage de transplantation.
Les vacances balnéaires s'inscrivent quasi totalementdans l'univers dece second type de voyage - un voyage où l'on se déplace non pour circuler: faire un tour, etexplorer le monde mais pour s'immobiliser et trouver un lieu de repli sur soi. Notons que le Club Mé́diterranée, dès ses origines, avait très bien perçu cette demande vacancière spécifique ${ }^{(6)}$, qui neréclame pas autre chose que la soustraction de l'individu hors du monde et non sa mise en contact avec l'extérieur.

C'est pourquoi, comme je l'ai déjà proposét , il me semble nécessaire de réhabiliter une distinction jadis bien établie, à savoir celle qui distingue le tourime de la villégiature - et cela non par simple minutie terminologique mais au titre de catégories pleinement opératoires permettant d'éviter des amalgames et l'usage de notions pléonastiques comme *tourisme de séjours ou *tourisme sédentaires.

Face à cette ampleur des vacances balnéaires, ellesembledésormais bien révolue cette affirmation de J. Dumazedier, qui, au début des années soixante, pronostiquant les effets de al'auto-boom» sur les loisirs, pouvait affirmer: *Le tourisme sédentaire diminue au profit du tourisme itinérants(s).

Certes, le concept même de *tourisme sédentaire» a profondément changé, dans la mesure où il ne réfere plus aujourd'hui, pour l'essentiel, à l'idée d'un retour chez soi (d'où, d'ailleurs, la chute en France des vacances à la campagne) ou à celle de vacances chez soi (selon les sources, le taux dedéparten vacances des Français fût de 59 $\%$ à $63 \%$ en 1990 , dont $54 \%$ à $55 \%$ en étê). Pour autant, ledit «tourisme sédentaires est bien loin d'avoir disparu et se maintient très largement sous une forme qui consiste non pas à retourner ou à rester chez soi mais, aux antipodes du dépaysenent, à aller en des lieux où lon se sent conme chez

Cette nuance psychologique n'est pas sans importance - tout au contraire. Elle est même essentielle, quoiqu'ayant été trop longtemps négligée dans l'analyse des comportements vacanciers - une analyse qui ne s'est pas attachée à distinguer clairementet à interroger cette différence, pourtant anthropologiquement fondamentale, qui détermine cette autre, entre circulation et migration: la différence entre voyage
Ainsi, notamment sous la forme de la villégiature balnéaire, le *tourisme sédentaires est bien loin d'avoir diminué et, a fortiori, d'être menacé de disparition ou, plus modestement, de régression sous les coups conjugués du développement du tourisme international (les séjoursà l'étranger furent de $11,1 \%$ en 1990 , dont, on l'a dit, $37 \%$ à la mer) ou du *tourisme verts (rural ou montagnard), qui n'est lui-même, le plus souvent qu'une autre forme de villégiature - à cette différence près que la *villégiature vertes a pour argument promotionnel majeur (sur le thème du retour à la terre) le *repaysements, alors que la *villégiature bleues: la balnéarité de masse, de par son espace et son aménagement, isolatexotique ou scène vide situés en marge du monde en dehors ou sur sa frange -, est $l e$ thêatre, litté alentent abstrait, d'une expérience vacancière d'une toute autre nature qui, culturellement parlant, ne relève ni du dépaysement ni du repaysement... (8)

Alors, pourquoi cette tendance lourde vers le littoral? Pourquoi cette ampleur du voyage vacancier de tramsplantation en direction du bord de mer? Et dès lors, quel avenir pour ce phénomène d'une incontestable importance, tant socio-culturelle qu'économique?

Cette tendance témoigne de la transformation profonde d'une sensibilité collective; ou, à tout le moins, de l'intensification d'une attitude de l'bomo vacans quant à son rapport au monde, aux loisirs et à l'envi- 
ronnement en général. Il est évidemment fort délicat d'apprécier exactement le contenu psychologique et affectif de cette transformation. Toutefois, quelques enquêtes qualitatives peuvent nous mettre sur la piste d'une première estimation.

Ily a, par exemple, constatéc ces dix dernières années, çe retour en force du sens de la famille. A l'affirmation: \&La famille est le seul endroit où l'on se sente bien et détendu $*, 61 \%$ des Français ont répondu positivement en 1982 ; et $69 \%$, en $1991 \%$. Or, quoique bien sûr elle n'en ait pas le monopole, la plage est en effet, de longue date, un des lieux privilégiés des vacances familiales: un territoire propice à la reconstitution de la «tribus et de la vie communautaire, ordinairement fragmentées ou dispersé́es le reste de l'année.

Comme l'écrivit J. Viard, «les rites de vacances ont permis une unification rếgếnérescente de ce que l'année de travail morcelle souvents ${ }^{(10)}$ - soulignant à cette occasion qu'en-deçà du thème de la découverte et de la rencontre de l'Autre (thème quelque peu surévalué par les spécialistes quant à son influence réelle sur les pratiques vacancières), les vacances sont peutetre d'abord, pour beaucoup d'entre nous, retrouvailles et rencontre du Même...

Toutefois, concernant les raisons de cette tendance lourde vers le littoral, ce beroin familial (même s'il trouve en ce lieu des conditions optimales de satisfaction) n'a rien de spécifique. Alors il y a le soleil? Certes, l'héliotropismeoriente à l'évidence les flux vacanciers; mais le soleil brille ailleurs! Et puis il y a aussi le tropisme marin; mais il faut se garder d'hypostasier celui-là comme un principe explicatif ràdical. Il ne peut que nous conduire à dire que l'on va au bord de la mer parce qu'il y a la mer!

Il y a encore d'autres mobiles, généraux ou spécifiques, à commencer par le désir de rupture ave la vie quotidienne. Pour $91 \%$ des personnes interrogées ${ }^{(11)}$, cette rupture est la définition mểme des vacances. Toutefois, les modalités de cette rupture sont diverses, selon qu'elle est touristique ou non, évasion ou repli sur soi, recherche du dépaysement, du repaysement ou bien ni l'un ni l'autre - ce qu'est la plage, un univers situé dans ce eni l'un ni l'autres, en ce qu'ellene propose au fond ni une décentration culturelle ni un retour aux sources, mais un intervalle de sociabilite abstraite.:-
Spécifique par contre, semble être cette donnée, à savoir que de toutes les destinations, la mer est de loin celle dont le choix est le plus ritualisé. En effet, $50 \%$ de la clientèle du bord de mer est une clientèle d'habitués, contre $30 \%$ pour la campagne et $15 \%$ seulement pour la montagne et l'étranger. Toutefois, concernant l'explication de l'attraction balnéaire, cette habitude: cette ritualité, est un effet, pas une cause.

Rendre compte des propriétés phénoménologiques spécifiques du site balnéaire apparaît donc ici plus que jamais indispensable; et on n'appréciera que davantagel'importance etla nécessité de cette exploration quand on saura que lechoixdes séjours et des hébergements par les Français est fait en fonction du lieu a $75 \%$ - contre $40 \%$ en fonction de la qualité et $25 \%$ en fonction des activités.

Ainsi, insistante, c'est bien la question du lieu plagique etde sa nature quirevient sans cesse. La plage n'est pas un *produits comme les autres et répond dece fait à une autre demande que celles, traditionnelles, de la découverte ou de l'immersion dans la natureetl'authenticité. Seulementwoilà, la plage est un lieu vacancier si évident, si habituel, qu'il n'en est que plus mystérieux - et son succès aussi, dont on s'étonne toujours à la vue de la foule qui l'investit.

Lieu abstrait, lieu de jouissance d'un *êtreensemble sans emplois (112), la plage, hors de toutes les réalités afférentes au quotidien d'origine, au naturel ou au contexte local, est en fait un espace où se stylise un dérir fondantental de regroupement en contrepoint d'un monde qui l'inhibe et rend son assouvissementproblématique-un monde, on le sait, générateur aujourd'hui de solitudes, d'éclatements, de déchirures et de conflits. Un monde qui brise des unités et des rythmes, des liens et des rites et qui en imposent d'autres. Unmonde, finalement, d'agressions et d'insécurités multiples.

Aussi, la quête del'Autre, del'Authentique, de la Nature, del'Indigène ou de l'Origine n'a guère sa place (sinon comme alibi) dans cet univers das de resocialisation idéale - un univers vacancier dont l'avenir n'est de ce fait en rien menacé et même plutôt radieux, tant que le monde *extérieur\% restera ce qu'il est, de plus en plus au demeurant, avec ses crises, ses menaces, ses contraintes, ses convulsions et ses désintégrations.
Il y a dans les vacances contemporaines du bord de mer - car ce n'était pas le cas au $\mathrm{XIX}^{e}$ siècle $^{(13)}$ - une recherche collective de sociabilité indépendante (*sans emploi») qui, même si elle s'effectue sur le mode du simulacre, rếpond précisément à un besoin d'abstraction communautaire bors du social concret, avec ses contingences matérielles, ses obligations relationnelles, ses justifications fonctionnellesetses règles anxiogènes.

De ce point de vue, la création et le succès des Center Parss en Europe (en Belgique, Hollande ou Grande-Bretagne) et en France ( 2 sont ouverts, 5 sont prévus pour l'an 2000) confirme la montée en puissance de ce besoin - le modèle Center Pars apparaissant à l'analyse, dans l'espace-temps des courts séjours etdes loisirs de proximité, comme un complément enclavé et condensé de ce qu'offre le bord de mer en été. De ce fait, Center Parcs explique la nature mêne del'attraction de la mer, dont il concentre les propriétés essentielles.

Car qu'est-ce qu'un Center Parci(14)? *Un microclimat méditerranéen sous une immense voûte transparentes, dit cette publicité - c'est-à-dire un univers balnéaire sous bulle, totalement artificialisé, où la piscine tropicale (avec palmiers, bananiers, cascades, vagues et tourbillons) a remplacé la mer; et la technique, les humeurs incontrôlables du soleil et des éléments. Ici aussi, et même plus que jamais, on n'a que fairede l'Authentique ou du Naturel. C'est encore de el'être-ensemble sans emplois que l'on propose et rend possible - une sociabilité hors du monde, hors des influences du milieu, y comprisles variations dimatiques. *29\% toute l'annéel $\$$ est d'ailleurs un des slogans publicitaires privilégiés du groupe.

Et comment est pensé un Center Parcs? Au niveau de l'espace, l'exemple de celui de Normandie (près de Verneuil-sur-Avre) est édifiant. Sur une superficie de 310 ha, 63 sont exploités, le reste protégeant le centre - un aménagement du site qui matérialise un besoin derupture qui n'estni de l'ordre de l'évasion ni de l'ordre du ressourcement, mais bien du détachement. Bref, un lieu de villégiature si indépendant del'extérieur quel'on ne peut décidément plus le ranger dans la catégorie des aloisirs touristiques $*$ ou de la *villégiature verte*, mêmes'il est implanté en pleine nature. Et au niveau de la clientèle - est-ce un hasard? -, c'est la famille, unité nucléaire de sociabilité, qui est visée et, de fait, attirée: $80 \%$ 
desvisiteurs ont aumoins un enfantet $56 \%$ plus d'un enfant de moins de quinze ans ${ }^{(15)}$.

Dès lors, et pour conclure, quels avenirs pour la mer? - dont on cerne mieux maintenant la nature des propriétés à l'origine de sa puissance d'attraction. Dans un contexte général d'insécurité et de désagrégation du lien social, qui incite au détachement et au repli sur soi, le bord de mer apparaît comme le haut-lieu de réparation et de compensation massives d'une carence de sociabilité. Affectée d'une telle valeur dans l'univers des loisirs, la mer ne peut donc que demeurer le territoire vacancier puissamment attractif qu'elle est déjà.

La prolifération (annoncée) des Center Paros pourrait toutefois pondérer cette prédiction, selon qu'ils rempliront dans le futur le rôle de compléments hivernaux au titre de lieux de séjours-relais entre vacances et quotidien ou bien le rôle de lieux vacanciers à part entière - ce qui est loin d'être exclu, suite à l'apparition d'un nouveau type devisiteur (non prévu par les dirigeants de Center Parcs eux-mêmes), qui préfere, à coût égal ou même supérieur, passer huit jours dans un centre plutôt que trois semaines sur une plage... $f$

Notes of ríférences

(1) G. Mermet, Francoscopie 1993, Paris, Larousse, 1992, p. 422

(2) Memento du tourisme, Paris, Observatoire National du Tourisme, 1991, p. 126. Source: INSEE, T.8. 15 .

(3) Le marche allemand th tourisme rural en France - étude qualitative rélisée auprès des professionnels et des consommateurs, remise au Commissariat au Plan -, Paris, D2X International, avnil 1990.

(4) P. Tsartas, ldentite et mabilite des deux groupes d'acteurs du tourisme en Grece: les touristes et less autochtones, communication non-publiee. colloque Le tourisme international entre tradition et modernițé, Nice, Carrefour Universitaire Mediterran\&en, novembre 1992.

(5) J. Dumazedier, Vers une eivillisation du loisir?. Paris, Le Seuil, 1972, p. 134 (1ère édition, 1962).

(6) C. Peyre et Y. Raynouard, Histoire of Ifgendes du Club Moditerranée, Paris, Le Seuil, 1971, p. 81 par exemple.

(7) J.D. Urbain, L'idiot du voyage, Paris, Plon, 1991, chapitre 1: Le touriste et les mots, p. 29 et suiv.

(6) Ce n'est pas le lieu ici de rendre compte dans le détail de cette singularite phénoménologique de l'expérience plagique. Précisons toutefois que l'auteur de ces lignes rédige en ce moment mëme un ouvrage quil traite précisément de l'espace balnéaire contemporain dans cette optique: des conditions historiques de production de cette "sçne vides, de son évolution esthétique et des propriétés sociales et symboliques spécifiques du lieu qui découlent de cet aménagement...

(9) Rapportépar G. Mermet, Francoscople 1993, op. cit., p. 126. Source; Paris, CREDOC (Centre de Recherche pour l"Etude et l"Observation des Conditions de vie).

(10) J. Viard, Penser lese vacances, Actes Sud, $H$ Nyssen Ed., 1984, p. 177.

(11) Ce résultat et ceux mentionnés d-après, communiqués par B. Préel lors d'une audition au Commissariat au Plan en juillet 1990, 6́manent d'une enquäte faite par le BIPE (Neuilly) aupres de 3000 personnes par entretiens individuels longs.

(12) M. Maffesoli, Le tempe des tribus. Le declin de Yindividualieme dane les weeibtós de masse. Paris, Méridien, Klincksieck et Cie, 1988, p. 121 et surv.

(13) A. Corbin, Le terrhtolre du vide. L'Occident et le dosir du rivage - 1750-1840, Paris, Aubier, 1988.

(14) Center Parcs est un groupe hollandais dont le siège central est à Fotterdam.

(15) Informations communiqués par M. Loeffen, directeur général de Center Parcs-Commissariat au Plan, Paris, octobre 1990. 